\title{
Real-Time Noise Reduction for Mössbauer Spectroscopy through Online Implementation of a Modified Kalman Filter
}

\author{
David G. Abrecht ${ }^{\mathrm{a}}$, Jon M. Schwantes ${ }^{\mathrm{a}}$, Ravi K. Kukkadapu ${ }^{\mathrm{b}}$, Benjamin S. McDonald ${ }^{\mathrm{a}}$, Gregory C. \\ Eiden $^{\mathrm{a}}$, Lucas E. Sweet ${ }^{\mathrm{a}}$ \\ ${ }^{a}$ National Security Directorate, Pacific Northwest National Laboratory, 902 Battelle Blvd., Richland, Washington, USA \\ 99352 \\ ${ }^{b}$ Environmental Molecular Sciences Laboratory, Pacific Northwest National Laboratory, 902 Battelle Blvd., Richland, \\ Washington, USA 99352
}

\begin{abstract}
Spectrum-processing software that incorporates a gaussian smoothing kernel within the statistics of first-order Kalman filtration has been developed to provide cross-channel spectral noise reduction for increased real-time signal-to-noise ratios for Mössbauer spectroscopy. The filter was optimized for the breadth of the gaussian using the Mössbauer spectrum of natural iron foil, and comparisons between the peak broadening, signalto-noise ratios, and shifts in the calculated hyperfine parameters are presented. The results of optimization give a maximum improvement in the signal-to-noise ratio of $51.1 \%$ over the unfiltered spectrum at a gaussian breadth of 27 channels, or $2.5 \%$ of the total spectrum width. The full-width half-maximum of the spectrum peaks showed an increase of $19.6 \%$ at this optimum point, indicating a relatively weak increase in the peak broadening relative to the signal enhancement, leading to an overall increase in the observable signal. Calculations of the hyperfine parameters showed no statistically significant deviations were introduced from the application of the filter, confirming the utility of this filter for spectroscopy applications.
\end{abstract}

Keywords: Mössbauer spectroscopy, software, spectrum filtering, signal-to-noise ratio

\section{Introduction}

Mössbauer spectroscopy is a resonant gamma ray absorption technique that has been highly successful for characterization of oxidation states, coordination environments, and magnetic properties of solid compounds in a variety of fields. The success of the technique in these applications is due to the narrow energy dispersion of the ${ }^{57} \mathrm{Fe}$ gamma ray, allowing extremely sensitive probing of hyperfine nuclear states created by electronic and magnetic structures within solid materials. Because of this sensitivity, properties such as the electric field gradient splitting of the nuclear states can be measured to a high degree of accuracy using simple benchtop instruments, making Mössbauer spectroscopy an extremely powerful tool for materials characterization [1].

Unfortunately, a drawback to this sensitivity, in practice, is a low count rate and low signal-to-noise ratio leading to typically long counting times for real samples. Research efforts in Mössbauer spectroscopy have often focused on mitigating these issues through clever instrument design and spectrum processing. Spectrum processing developments have primarily been directed at post-processing of the spectrum to obtain exact values of the hyperfine parameters, beginning with simple deconvolution methods represented by the work of Stone [2], and moving into automation and more advanced computational methods to improve the fits to the

\footnotetext{
${ }^{*}$ Corresponding author

Email address: david.abrecht@pnnl.gov (David G. Abrecht)
} 
spectra [3], including the recent inclusion of genetic and evolution algorithms [4,5] and database matching techniques [6]. Efforts to improve the sensitivity and speed of measurements have historically focused on hardware developments, such as the development of capillary gamma ray lenses [7], detector and electronics improvements [8], and the use of synchrotron radiation [9].

Speed and sensitivity improvements have been achieved through software applications in other forms of optical, magnetic, and electronic spectroscopy[10, 11]. Smoothing algorithms such as Savitzky-Golay [12] and Whittaker [13] smoothers are common for extracting peaks from noisy data, and are available in commercial spectrum processing software [14]. However, such techniques are often discouraged for Mössbauer spectroscopy due to an undesirable broadening of the peaks caused by these smoothers and the common presence of highly overlapping peaks within the spectra [15, 16]. Advancements in processing power and memory management, however, have enabled researchers to revisit software algorithms as a means of improving spectrum characteristics, leading to improvements in the speed and operations of Mössbauer spectroscopy beyond what was available from hardware improvements alone. The use of pulse processing [17] has been shown to improve linewidths and count rates, and modified algorithms for communications, digital timing, and control of the drive have been shown to decrease deadtimes and non-linearities $[18,19]$. Despite these successes, few attempts to improve counting times by extracting lineshapes from the background, similar to the smoothing algorithms, have been documented. In this work, we describe such a spectral processing technique for Mössbauer spectroscopy that operates in real-time, based on the application of a modified Kalman filter, and discuss the effects of the filter application on the spectral shape and hyperfine parameters.

\section{Description of the Kalman Filter}

The Kalman filter is a predictor-corrector type filter that is commonly employed in the aerospace and robotics industry for computer navigation and signal extraction [20]. Based on the original work by Kalman and Bucy $[21,22]$, these filters utilize estimates of the noise and process covariance matrices to optimize the signal response based on a known model for the time evolution of a system under study. In the general Kalman filter, for the set of $n$ measurements of a process state, $\boldsymbol{z}_{\mathrm{i}}$, described by $r$ state properties, $\boldsymbol{x}_{\mathrm{i}}$, where $i$ represents the current time step, the measurements as a function of the state of the process can be described by

$$
z_{\mathrm{i}}=\boldsymbol{H}_{\mathrm{i}} \boldsymbol{x}_{\mathrm{i}}+\boldsymbol{v}_{\mathrm{i}}
$$

where $\boldsymbol{H}_{\mathrm{i}}$ is an $n \times r$ matrix describing the dependence of the measurements on the state properties and $\boldsymbol{v}_{\mathrm{i}}$ is an $n$ length vector describing the noise associated with the measurements. A prediction of the next state of the system can then be made, given by

$$
\boldsymbol{x}_{\mathrm{i}+1}=\boldsymbol{\Phi}_{\mathrm{i}} \boldsymbol{x}_{\mathrm{i}}+\boldsymbol{u}_{\mathrm{i}}
$$

where $\boldsymbol{\Phi}_{\mathrm{i}}$ is an $r \times r$ matrix representing the expected dynamic behavior of the system and $\boldsymbol{u}_{\mathrm{i}}$ is an $r$ length vector describing the process noise. Both $\boldsymbol{u}_{\mathrm{i}}$ and $\boldsymbol{v}_{\mathrm{i}}$ are assumed to be uncorrelated zero-mean Gaussian white noise sequences with covariance matrices $\boldsymbol{Q}$ and $\boldsymbol{R}$, respectively. A set of predictions of the process state, $\hat{x}_{\mathrm{i}}$, is then obtained from the measurements $\boldsymbol{z}_{\mathrm{i}}$ and optimized by iterative solution of the Ricatti equations, with the iteration and optimization given by

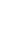

$$
\begin{gathered}
\hat{\boldsymbol{x}}_{\mathrm{i}}^{\circ}=\boldsymbol{\Phi}_{\mathrm{i}} \hat{\boldsymbol{x}}_{\mathrm{i}} \\
\boldsymbol{M}_{\mathrm{i}}=\boldsymbol{\Phi}_{\mathrm{i}} \boldsymbol{P}_{\mathrm{i}} \Phi_{\mathrm{i}}^{T}+\boldsymbol{Q} \\
\boldsymbol{K}_{\mathrm{i}}=\boldsymbol{M}_{\mathrm{i}} \boldsymbol{H}_{\mathrm{i}}^{T}\left[\boldsymbol{H}_{\mathrm{i}} \boldsymbol{M}_{\mathrm{i}} \boldsymbol{H}_{\mathrm{i}}^{T}+\boldsymbol{R}\right]^{-1}
\end{gathered}
$$




$$
\begin{gathered}
\hat{\boldsymbol{x}}_{\mathrm{i}+1}=\hat{\boldsymbol{x}}_{\mathrm{i}}^{\circ}+\boldsymbol{K}_{\mathrm{i}}\left[\boldsymbol{z}_{\mathrm{i}}-\boldsymbol{H}_{i} \hat{\boldsymbol{x}}_{\mathrm{i}}^{\circ}\right] \\
\boldsymbol{P}_{\mathrm{i}+1}=\left[\boldsymbol{I}^{r}-\boldsymbol{K}_{\mathrm{i}} \boldsymbol{H}_{\mathrm{i}}\right] \boldsymbol{M}_{\mathrm{i}}
\end{gathered}
$$

where $\boldsymbol{P}_{\mathrm{i}}$ is the $r \times r$ covariance matrix of the error between the predicted and actual process states at time $i, \boldsymbol{K}_{\mathrm{i}}$ is the current step filter gain $r \times n$ matrix, $\boldsymbol{I}^{r}$ is the $r \times r$ identity matrix, and the -1 and $T$ superscripts represent the matrix inverse and transpose, respectively. A more detailed description of the derivation of the general filter are given by Kalman [21], Kalman and Bucy [22], Mehra [23], and Bélanger [24]. The five equations above correspond to the five operations of the filter. In Equation 1a, a prediction of the next state is calculated based on the user-supplied model and the filtered values from the previous step. Equation $1 \mathrm{~b}$ then calculates the covariance of the predicted state, and adds the covariance of the signal noise given by $\boldsymbol{Q}$. The filter gain, $\boldsymbol{K}_{\mathrm{i}}$, is then calculated by Equation 1c, which is optimized to minimize the difference between the predicted and 'actual' states of the system if $\boldsymbol{Q}$ and $\boldsymbol{R}$ are known exactly and $\boldsymbol{\Phi}_{\mathrm{i}}$ is an accurate model of the system. The prediction is then corrected by the difference between the prediction and the data, multiplied by the gain, to give the new filtered state in Equation 1d. Finally, Equation 1e calculates the new covariance matrix for the filtered state for calculation in the next iteration.

In the application of the filter, the covariance matrix $\boldsymbol{P}_{\mathrm{i}}$ and gain $\boldsymbol{K}_{\mathrm{i}}$ are solved by the iteration and should converge after relatively few steps. The filter requires that a user specify $\boldsymbol{H}_{\mathrm{i}}$ and $\boldsymbol{\Phi}_{\mathrm{i}}$ as the model for the process. The initial values of $\boldsymbol{P}_{0}$ and $\hat{\boldsymbol{x}}_{0}$ are also specified by the user, although the filter convergence has been shown not to depend highly on these starting values [20]. The values for $\boldsymbol{Q}$ and $\boldsymbol{R}$ are user-specified parameters for the model, and can be obtained either through trial and error or through offline testing of the noise responses, although adaptive methods are also available [23, 24].

\section{Mössbauer Process Model}

To apply a Kalman filter to a process, a suitable model of the measurement process must be developed. For a spectrometer, this requires an appropriate description of how the detector is operated, and how the operation correlates to the expected process being measured. In Mössbauer spectrometry, the detector and software accumulate counts and develop the spectrum based on the relative differences in gamma ray flux as a function of time, as correlated to the time-dependent Doppler velocity applied to the source. To develop a Kalman filter around this process, each channel of the spectrum was considered to be a single 'bin' into which a constant gamma ray flux passed during each measurement interval. The counts on each channel were then modeled as the integration of that constant flux, i.e. a linear function of time. For a hypothetical five-channel spectrum, the model equation components describing this behavior would be 


$$
\begin{aligned}
& \hat{\boldsymbol{x}}_{\mathrm{i}}=\left[\begin{array}{c}
\hat{x}_{1} \\
\hat{x}_{2} \\
\hat{x}_{3} \\
\hat{x}_{4} \\
\hat{x}_{5} \\
\frac{\mathrm{d} \hat{x}_{1}}{\mathrm{~d} t} \\
\frac{\mathrm{d} \hat{x}_{2}}{\mathrm{~d} t} \\
\frac{\mathrm{d} \hat{x}_{3}}{\mathrm{~d} t} \\
\frac{\mathrm{d} \hat{x}_{4}}{\mathrm{~d} t} \\
\frac{\mathrm{d} \hat{x}_{5}}{\mathrm{~d} t}
\end{array}\right]_{\mathrm{i}} \quad \hat{z}_{\mathrm{i}}=\left[\begin{array}{c}
z_{1} \\
z_{2} \\
z_{3} \\
z_{4} \\
z_{5}
\end{array}\right]_{\mathrm{i}} \\
& \boldsymbol{H}_{\mathrm{i}}=\left[\begin{array}{llllllllll}
1 & 0 & 0 & 0 & 0 & 0 & 0 & 0 & 0 & 0 \\
0 & 1 & 0 & 0 & 0 & 0 & 0 & 0 & 0 & 0 \\
0 & 0 & 1 & 0 & 0 & 0 & 0 & 0 & 0 & 0 \\
0 & 0 & 0 & 1 & 0 & 0 & 0 & 0 & 0 & 0 \\
0 & 0 & 0 & 0 & 1 & 0 & 0 & 0 & 0 & 0
\end{array}\right]_{\mathrm{i}}, \quad \forall i, \\
& \boldsymbol{\Phi}_{\mathrm{i}}=\left[\begin{array}{llllllllll}
1 & 0 & 0 & 0 & 0 & t & 0 & 0 & 0 & 0 \\
0 & 1 & 0 & 0 & 0 & 0 & t & 0 & 0 & 0 \\
0 & 0 & 1 & 0 & 0 & 0 & 0 & t & 0 & 0 \\
0 & 0 & 0 & 1 & 0 & 0 & 0 & 0 & t & 0 \\
0 & 0 & 0 & 0 & 1 & 0 & 0 & 0 & 0 & t \\
0 & 0 & 0 & 0 & 0 & 1 & 0 & 0 & 0 & 0 \\
0 & 0 & 0 & 0 & 0 & 0 & 1 & 0 & 0 & 0 \\
0 & 0 & 0 & 0 & 0 & 0 & 0 & 1 & 0 & 0 \\
0 & 0 & 0 & 0 & 0 & 0 & 0 & 0 & 1 & 0 \\
0 & 0 & 0 & 0 & 0 & 0 & 0 & 0 & 0 & 1
\end{array}\right]_{\mathrm{i}}, \forall i
\end{aligned}
$$

where $t$ is the time interval between measurements. This model utilized within the Kalman filter provides noise reduction within each channel with respect to time, but each channel remains independent and interchannel noise is still present. In order to produce interchannel noise reduction, each channel must have a dependence on the neighboring channels introduced into the Kalman filter statistics. In this work, this dependency was introduced by convoluting the channel states, but not the derivatives, with a normalized gaussian smoothing kernel given by the equation

$$
g(x)=\frac{1}{\sqrt{\sigma \pi}} e^{\frac{-(x-c)^{2}}{\sigma}}
$$

\footnotetext{
where $x$ is channel number, $c$ is the central channel number for which this value is calculated, and $\sigma$ is a user-defined parameter used to control the breadth of the convolution. For the example given in Equation 2 , a convolution truncated over three channels with $\sigma=1$ would modify $\boldsymbol{\Phi}_{\mathrm{i}}$ to give
} 


$\mathbf{\Phi}_{\mathrm{i}}=\left[\begin{array}{cccccccccc}0.5642 & 0.2076 & 0 & 0 & 0.2076 & 0.5642 t & 0.2076 t & 0 & 0 & 0.2076 t \\ 0.2076 & 0.5642 & 0.2076 & 0 & 0 & 0.2076 t & 0.5642 t & 0.2076 t & 0 & 0 \\ 0 & 0.2076 & 0.5642 & 0.2076 & 0 & 0 & 0.2076 t & 0.5642 t & 0.2076 t & 0 \\ 0 & 0 & 0.2076 & 0.5642 & 0.2076 & 0 & 0 & 0.2076 t & 0.5642 t & 0.2076 t \\ 0.2076 & 0 & 0 & 0.2076 & 0.5642 & 0.2076 t & 0 & 0 & 0.2076 t & 0.5642 t \\ 0 & 0 & 0 & 0 & 0 & 1 & 0 & 0 & 0 & 0 \\ 0 & 0 & 0 & 0 & 0 & 0 & 1 & 0 & 0 & 0 \\ 0 & 0 & 0 & 0 & 0 & 0 & 0 & 1 & 0 & 0 \\ 0 & 0 & 0 & 0 & 0 & 0 & 0 & 0 & 1 & 0 \\ 0 & 0 & 0 & 0 & 0 & 0 & 0 & 0 & 0 & 1\end{array}\right]_{\mathrm{i}} \quad \forall i$

where the spectrum is wrapped if the convolution extends beyond the last channel. For an actual Mössbauer spectrum of 1024 channels, the examples given in Equations 2, 3, and 4 are extended to $\mathrm{n}=1024$ channels, $r=2048$, to create the entire spectrum.

\section{Experimental}

To test the application of the filter, ${ }^{57} \mathrm{Fe}$ Mössbauer spectra were collected from a $27.3 \mu \mathrm{m}$ isotopicallynatural body centered cubic $\alpha$-iron foil at room temperature with a 1024 channel See Co. model W302 Resonance Gamma Ray spectrometer in the transmission configuration using a ${ }^{57} \mathrm{Co}$ in Rh source and an Amptek XR-100T CdTe solid-state gamma ray detector collimated with a $400 \mu \mathrm{m}$ aperture. Iron foil used in the experiments was provided by See Co.

Measurements and Kalman filter operations were performed on a desktop PC running Windows 7 with an Intel i5-2400 $3.10 \mathrm{GHz}$ quad core processor and 6.00 GB installed RAM. Three cores were utilized for the filter operations, and the remaining core was used for the Mössbauer instrument operations. Kalman filters utilizing a gaussian smoothing function, as exemplified in Equations 2 and 4, were applied at 2 minute intervals to the unfolded data in real time as it was acquired from the spectrometer. Gaussian convolution kernels were truncated below $5.0 \times 10^{-6}$. Filtered and unfiltered data were acquired in parallel every 20 minutes up to 1400 minutes $(i=700)$ total spectrum collection time. Measurements were taken for selected values of $\sigma$ within the range $\sigma=2$ to $\sigma=262.5$, representing smoothing across 9 channels and 93 channels, respectively, to optimize the gaussian kernel.

For all runs, the initial matrix $\boldsymbol{P}_{0}$ was chosen such that all elements were equal to 1. Values for the elements of the initial predictor vector $\hat{\boldsymbol{x}}_{0}$ were obtained from the first measurement dataset. The values of the noise estimates $\boldsymbol{Q}$ and $\boldsymbol{R}$ were intentionally detuned, such that

$$
\boldsymbol{Q}=q\left[\begin{array}{cc}
\boldsymbol{I}^{n} & \mathbf{0} \\
\mathbf{0} & \mathbf{0}
\end{array}\right], \quad \boldsymbol{R}=r \boldsymbol{I}^{n}
$$

where $\mathbf{0}$ is an $n \times n$ matrix where all elements have a value of zero and the constants $q$ and $r$ were chosen to have a value of 100 for the purposes of this work. Although this detuning ensures that the optimum noise reduction is not obtained for the spectrum, this set of estimates for $\boldsymbol{Q}$ and $\boldsymbol{R}$ provide practical benefits in the functional form of the Ricatti equations given in Equation 1. Combined with the symmetric initial values $\boldsymbol{P}_{0}$, these modifications ensure that the matrices $\boldsymbol{M}_{\mathrm{i}}, \boldsymbol{K}_{\mathrm{i}}$, and $\boldsymbol{P}_{\mathrm{i}}$ remain symmetric or block symmetric throughout all iterations and several of the matrix operations in Equation 1 are commutative, greatly enhancing the speed of matrix operations within the code and enabling real-time application of the filter. Future work to incorporate more optimal estimates for $\boldsymbol{Q}$ and $\boldsymbol{R}$ would provide additional noise reduction benefits and would be enabled by improved processing capability. 
Filtered and unfiltered data were folded by an algorithm designed to minimize the sum of differences between the first half of the spectrum and the inverted second half shifted by any number of channels. Channel velocity was calibrated in all measurement runs against the unfiltered spectrum of the iron foil for that run after 1400 minutes. Isomer shifts were calculated as the average of all six peak positions, referenced to the source. The hyperfine magnetic field was calculated by the difference of the positions of peak pairs divided by $\mu_{\mathrm{N}}\left(\frac{\mu_{\text {exc }}}{I_{\text {exc }}}-\frac{\mu_{\mathrm{g}}}{I_{\mathrm{g}}}\right)$, where $\mu_{\mathrm{N}}$ is the nuclear magneton in $\mathrm{eV} / \mathrm{T}, \mu_{\mathrm{exc}}$ is the excited state nuclear moment in nuclear magnetons, $\mu_{\mathrm{g}}$ is the ground state nuclear moment in nuclear magnetons, and $I_{\mathrm{exc}}$ and $I_{\mathrm{g}}$ are the excited and ground state nuclear spins, respectively. The results from each of the three peaks were averaged to obtain the final number. The principle component of the electric field gradient, $V_{\mathrm{zz}}$, was then solved from each peak position, and the average of the six peaks was used to obtain the final value. The electric quadrupole splitting (EQS) was then obtained as $\frac{1}{4} e Q V_{\mathrm{zz}}$, where $Q$ is the nuclear quadrupole moment and e is the electron charge.

To calculate signal and noise intensity, a constant background plus six Lorentzian peaks of the form

$$
L\left(\beta, \Gamma, x, x_{0}\right)=\frac{\beta \Gamma}{\left(x-x_{0}\right)^{2}+\Gamma^{2}}
$$

were fitted simultaneously to the iron foil spectra, where $\beta$ and $\Gamma$ are variables describing the intensity and breadth of the Lorentzian, respectively, $x_{0}$ is the centroid position of the Lorentzian, and $x$ is the velocity in $\mathrm{mm} / \mathrm{s}$. Filtered and unfiltered spectra were fit independently. Inner, middle, and outer peak pairs were fit using single values of $\beta$ and $\Gamma$ for each pair. The centroid positions, $x_{0}$, were fit to the 1400 minute spectra and were held constant at these values for spectra at all other times within a run in order to minimize variations in the calculated Mössbauer parameters due to uncertainty in the fits. Signal intensity (SI) and full-width half-maximum (FWHM) of the innermost peak pair were then calculated as

$$
\mathrm{SI}=\frac{\beta}{\pi \Gamma}, \quad \quad \mathrm{FWHM}=2 \Gamma
$$

Background noise intensity (NI) was calculated as the root-mean square average of the difference between the fitted spectrum and the data. Signal-to-noise ratios (SNR) were then calculated for the innermost peak pair as the ratio SI/NI for each collected spectrum. These values were fit for filtered and unfiltered data for each run to the equation

$$
\mathrm{SNR}=a(i)^{\frac{1}{2}}
$$

where $a$ is the fitted parameter. The final ratio $\zeta=a_{\text {filtered }} / a_{\text {unfiltered }}$ was then calculated to evaluate the noise reduction due to the filter.

\section{Results and Discussion}

Typical results of the Kalman filter after 700 applications appear in green in Figure 1 for gaussian smoothing kernels given $\sigma=16.5$. These values correspond to normalized smoothing over 27 and 93 channels, respectively. In addition, the results of a post-processing convolution of the final raw spectrum with the same gaussian smoothing kernel appears in orange, for comparison. Qualitatively, the filter provides a reduction in the noise intensity along the background relative to the unfiltered data, without changing the direction of the deviation from the baseline for each channel or producing new artifacts in the spectrum. This behavior is in contrast to the behavior of the post-process gaussian smoother, which averages the final spectrum across nearest neighbor points and can create situations where the channel intensity is inverted relative to the 
average and can create "false peaks" in the spectrum, as shown clearly between 5 and $10 \mathrm{~mm} / \mathrm{s}$ in Figure 1. The change in behavior is due to the incorporation of the gaussian convolution into $\boldsymbol{\Phi}_{\mathrm{i}}$, which allows the smoothing to be included in the statistics of the Kalman filter and corrected by the original data given by Equation 1d. The incorporation allows smoothed noise to partially recover, and prevents the repeated application of a gaussian smoother from completely removing the spectrum peaks or accentuating noise.

Quantitatively, the improvement in the SNR can be determined through calculation of the $a$ parameters in Equation 5 and calculation of the filter enhancement ratio $\zeta=a_{\text {filtered }} / a_{\text {unfiltered. }}$ An example of these fits for a filter with $\sigma=4$ is shown in Figure 2. For both filtered and unfiltered data, the SNRs were found to follow the typical power law behavior with count times, although deviations at early times were observed because of the increased uncertainty in fitting peaks at low counts. FWHM values for both filtered and unfiltered spectra peaks were found to remain relatively constant throughout the run, with a similar increase at low counting times due to uncertainties in the fits.

Figure 3 shows the variation in $\zeta$ with respect to the extent of broadening of the gaussian kernel. The optimum value of $\zeta$ was found to occur at $\sigma=16.5$, which corresponds to smoothing across 27 channels. At $\sigma$ $<16.5, \zeta$ increases rapidly, corresponding to increasingly broad gaussian functions and increased cross-channel communication. The filter character in this region is dominated by the averaging of the high-frequency noise, which is expected to be zero-mean. This leads to a rapid increase in the SNR as noise is removed from the system. At $\sigma>16.5$, the $\zeta$ values slowly asymptote to $\zeta=1$, where no improvement in the SNR is obtained. In the high $\sigma$ region, the slow asymptote is dominated by the increased broadening and reduction of intensity of the main peaks. The high-frequency noise character under these conditions is eliminated by the gaussian smoother at each iteration by averaging over a large number of neighbors, and is only partially recovered by the filter through Equation 1d. This behavior creates a steady-state for the relative RMS noise intensity at high $\sigma$ values, keeping the noise intensity relatively constant for increasing values of $\sigma$. The reduction in the signal intensity due to broadening is thus the primary cause of the reduction in $\zeta$ in this range, but the reduction is slowed by compensation from increases in the second term correction in Equation 1d. At the highest values, the elements of the predictor $\hat{\boldsymbol{x}}_{i}^{\circ}$ create a nearly flat spectrum, and the deviation between the measured data and the predictor becomes large, dominating the calculation of $\hat{\boldsymbol{x}}_{i+1}$ and leading to an asymptotic approach to $\zeta=1$, a scaled reproduction of the original measurements $\boldsymbol{z}_{\mathrm{i}}$ and their empirical derivatives for the corrected state. The maximum at approximately $\sigma=16.5$ represents the best balance between the averaging of the noise peaks and the averaging of the signal peaks. The dependence of this maximum point on the noise frequency and original peak breadth has not yet been tested in this study but would be useful to examine in future work aimed at employing these filters.

In addition, Figure 3 shows the variation in the FWHM with $\sigma$, and the ratio $\epsilon=\zeta / F W H M$ for both the Kalman filter and post-process gaussian smoothing. The FWHM of the filtered peaks increases relatively steadily until reaching a plateau at approximately $\sigma=65$. At values greater than $\sigma=65$, the FWHM continues to increase but at a reduced rate, which is the primary factor for improvement relative to postprocess smoothing in this region. As with the noise, this occurs because the gaussian kernel effectively eliminates the majority of the signal intensity at these values, but the correction in Equation 1d partially restores the peak shape. The ratio $\epsilon$ represents the tradeoff between noise reduction and peak broadening. A maximum in this ratio was not found, but rather increased continuously as $\sigma$ is decreased. A logarithmic expression was found to produce a good fit to the behavior, such that

$$
\epsilon=-0.138442 \ln (\sigma)+1.52729 .
$$

For spectroscopy applications, it is critical that the centroid positions of the peaks not be altered by the filter. In Mössbauer spectroscopy, any such alterations would appear as a change in the values of the calculated isomer shift (IS), electric quadrupole splitting (EQS), and hyperfine magnetic field (HMF) hyperfine parameters. A comparison between the filtered and unfiltered values for these hyperfine parameters for the Kalman filters applied in this work are given in Table 1. As shown in the table, shifts in the values of the hyperfine parameters for iron foil due to the filter fall within two standard deviations of the values derived 
from the peak positions of the unfiltered values, indicating that the filter does not produce a statistically significant shift in the hyperfine parameters for iron foil.

\section{Conclusions}

In this work, we have introduced a new software filter for real-time improvement in the signal-to-noise ratio of acquired Mössbauer spectra, based on Kalman filtration algorithms common in computer navigation. The filter was shown to improve signal-to-noise ratios by up to $51.1 \%$, with parameter-controllable levels of peak broadening. Further, the characteristics of the filter prevent the signal-to-noise ratio from becoming less than that of the unfiltered spectrum, although poor tuning can lead to excessive broadening. Filtered spectra showed no significant deviation in the derived hyperfine parameters, indicating the utility of the filter for spectroscopic applications. Future work to investigate the frequency dependence of the filter parameters and improvements in computational power would allow additional improvements in the noise estimates to be implemented, leading to improved filter performance and greater utility for Mössbauer spectroscopy as a materials characterization tool.

\section{Acknowledgements}

The authors would like to thank Drs. Sean Robinson and Rick Wittman for insightful comments and indepth review. We would also like to acknowledge the Environmental Molecular Sciences Laboratory (EMSL), located at Pacific Northwest National Laboratory, for assistance with the Mössbauer efforts. This work was funded by Pacific Northwest National Laboratory utilizing Laboratory Directed Research and Development (LDRD) funds. 
[1] B. Fultz, "Mössbauer Spectrometry," in Characterization of Materials. Elton Kaufmann, Editor (John Wiley, New York, 2011).

[2] A. J. Stone, Chemical Physics Letters 6 (1970) 331.

[3] P. A. de Souza Jr., Hyperfine Interactions 113 (1998) 383.

[4] Z. Klencsár, Nuclear Instruments and Methods in Physical Research B 129 (1997) 527.

[5] H. Ahonen, P. A. de Souza Jr., V. K. Garg, Nuclear Instruments and Methods in Physical Research B 124 (1997) 633.

[6] Z. Klencsár, Hyperfine Interactions 217 (2013) 117.

[7] Y. Yoshida, K. Suzuki, K. Hayakama, K. Yukihira, H. Soejima, Hyperfine Interactions 188 (2009) 121.

[8] D. Jancik, M. Mashlan, R. Zboril, J. Adetunji, K. Nomura, Czechoslovak Journal of Physics 55 (2005) 803.

[9] L. Yan, J. Zhao, T. S. Toellner, R. Divan, S. Xu, Z. Cai, J. S. Boesenberg, J. M. Friedrich, S. P. Cramer, E. E. Alp, Journal of Synchrotron Radiation 19 (2012) 814.

[10] S. Evans and A. G. Hiorns, Surface and Interface Analysis 8 (1986) 71.

[11] P. H. C. Eilers, Analytical Chemistry 75 (2003) 3631.

[12] A. Savitzky, M. J. E. Golay, Analytical Chemistry 36 (1964) 1627.

[13] E. T. Whittaker, Edinburgh Mathematical Society 41 (1923) 63.

[14] M. A. Bernstein, S. Sýkora, C. Peng, A. Barba, C. Cobas, Analytical Chemistry 85 (2013) 5778.

[15] T. S. Peretyazhko, J. M. Zachara, R. K. Kukkadapu, S. M. Heald, I. V. Kutnyakov, C. T. Resch, B. W. Arey, C. M. Wang, L. Kovarik, J. L. Phillips, D. A. Moore, Geochimica et Cosmochimica Acta 92 (2012) 48.

[16] D. G. Abrecht, J. A. Muñoz, H. L. Smith, B. Fultz, Journal of Physical Chemistry C 118 (2014) 1783.

[17] P. Novak, J. Navarik, J. Pechousek, V. Prochazka, L. Machala, J. Tucek, Journal of Instrumentation 9 (2014) T01001.

[18] J. Pechoušek, P. Novák, J. Navařík, P. Kohout, L. Machala, Journal of Electrical Engineering 64 (2013), 386.

[19] J. Pechousek, R. Prochazka, M. Mashlan, D. Jancik, J. Frydrych, Measurement Science and Technology 20 (2009) 017001.

[20] P. Zarchan, H. Musoff, Fundamentals of Kalman Filtering: A Practical Approach. 2nd ed. Lexington, MA American Institute of Aeronautics and Astronautics; 2005.

[21] R. E. Kalman, Transactions of the ASME-Journal of Basic Engineering 82 (1960) 35.

[22] R. E. Kalman, R. S. Bucy, Journal of Basic Engineering 83 (1961) 95.

[23] R. K. Mehra, IEEE Transactions on Automatic Control 17 (1972) 693.

[24] P. R. Bélanger, Automatica 10 (1974) 267. 
Table 1: Isomer Shift (IS), Electric Quadrupole Splitting (EQS), and Hyperfine Magnetic Field (HMF) calculated from the filtered and unfiltered iron foil experiments for all values of $\sigma$. Asymmetry was not considered in calculation of EQS. IS and EQS are given in units of $\mathrm{mm} / \mathrm{s}$, and HMF is given in units of tesla

\begin{tabular}{|c|c|c|c|c|c|c|}
\hline \multirow[b]{2}{*}{$\sigma$} & \multicolumn{3}{|c|}{ Run 1} & \multicolumn{3}{|c|}{ Run 2} \\
\hline & IS & EQS & $\mathrm{HMF}$ & IS & EQS & HMF \\
\hline 262.5 & -0.00460 & 0.00584 & 33.0191 & -0.00941 & 0.00151 & 32.9674 \\
\hline 131 & -0.00789 & 0.00583 & 32.7333 & -0.00391 & 0.00434 & 33.0147 \\
\hline 65.5 & -0.00225 & 0.00189 & 32.9793 & -0.00600 & 0.00102 & 32.9387 \\
\hline 33 & -0.00339 & 0.00340 & 32.8654 & 0.00073 & -0.00344 & 32.8724 \\
\hline 16.5 & -0.01413 & 0.00345 & 32.8981 & -0.00686 & 0.00968 & 33.0321 \\
\hline 8 & -0.00746 & 0.00600 & 32.7892 & -0.00623 & 0.00224 & 32.9543 \\
\hline 4 & -0.00148 & -0.00309 & 32.8067 & -0.00899 & 0.00856 & 33.1483 \\
\hline 2 & -0.00600 & 0.00573 & 33.0417 & -0.00749 & 0.00003 & 32.8655 \\
\hline unfiltered & -0.00650 & 0.00462 & 32.9461 & & & \\
\hline std. dev., unfiltered & 0.00358 & 0.00461 & 0.0657 & & & \\
\hline
\end{tabular}




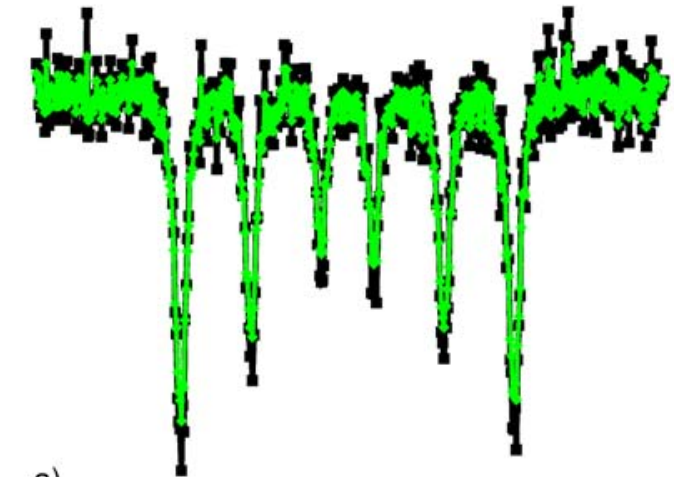

a)
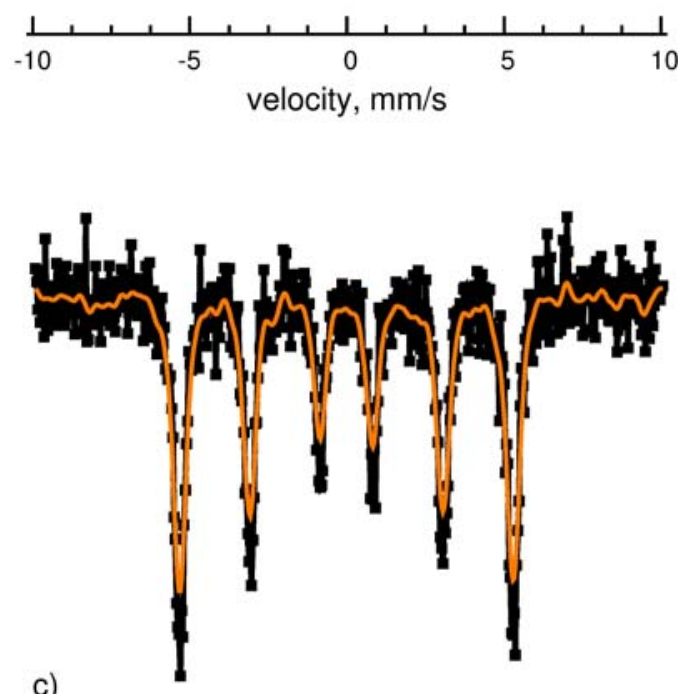

c)

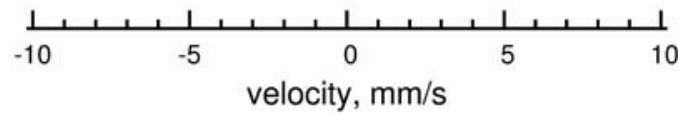

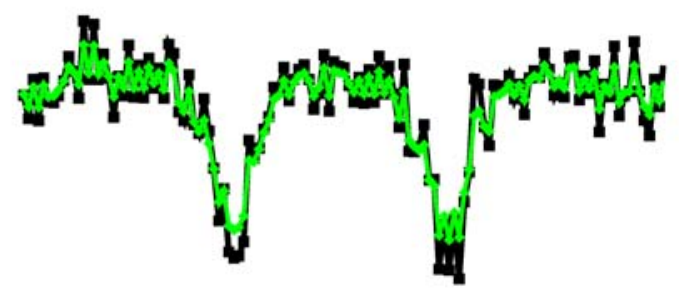

b)
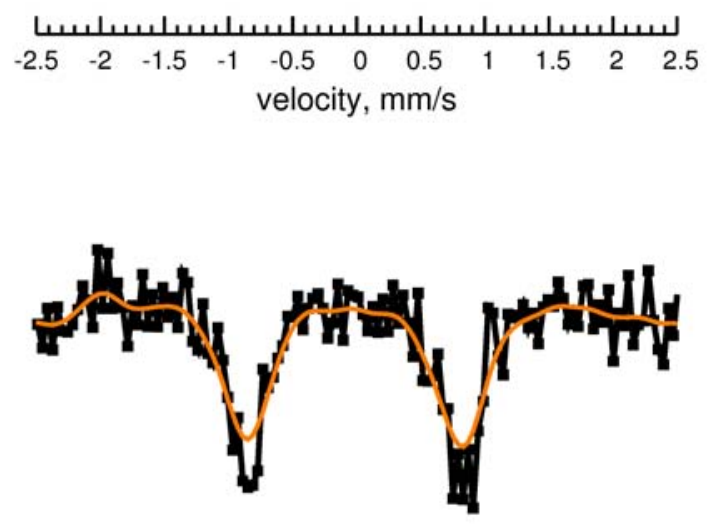

d)

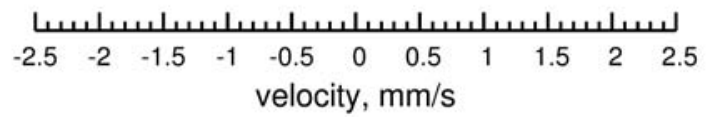

Figure 1: Comparison of unfiltered (black), filtered (green) and post-process smoothed (orange) spectra for iron foil. The filtered spectrum uses the Kalman filter with a gaussian smoothing kernel with $\sigma=16.5$, shown for the full spectral width (a) and in detail for the inner peaks (b). The smoothed spectrum was acquired by convoluting the final raw spectrum with the same gaussian kernel used in the Kalman filter, and similar views relative to the raw spectrum are shown in c) and d). The filtered data demonstrates a reduced background noise intensity without alterating the noise direction on individual channels, relative to the unfiltered data. The smoothed spectra shows greater noise reduction, but significantly increases peak FWHM and introduces artifact peaks from convolution of the noise, as demonstrated at $-4.25 \mathrm{~mm} / \mathrm{s}$. 


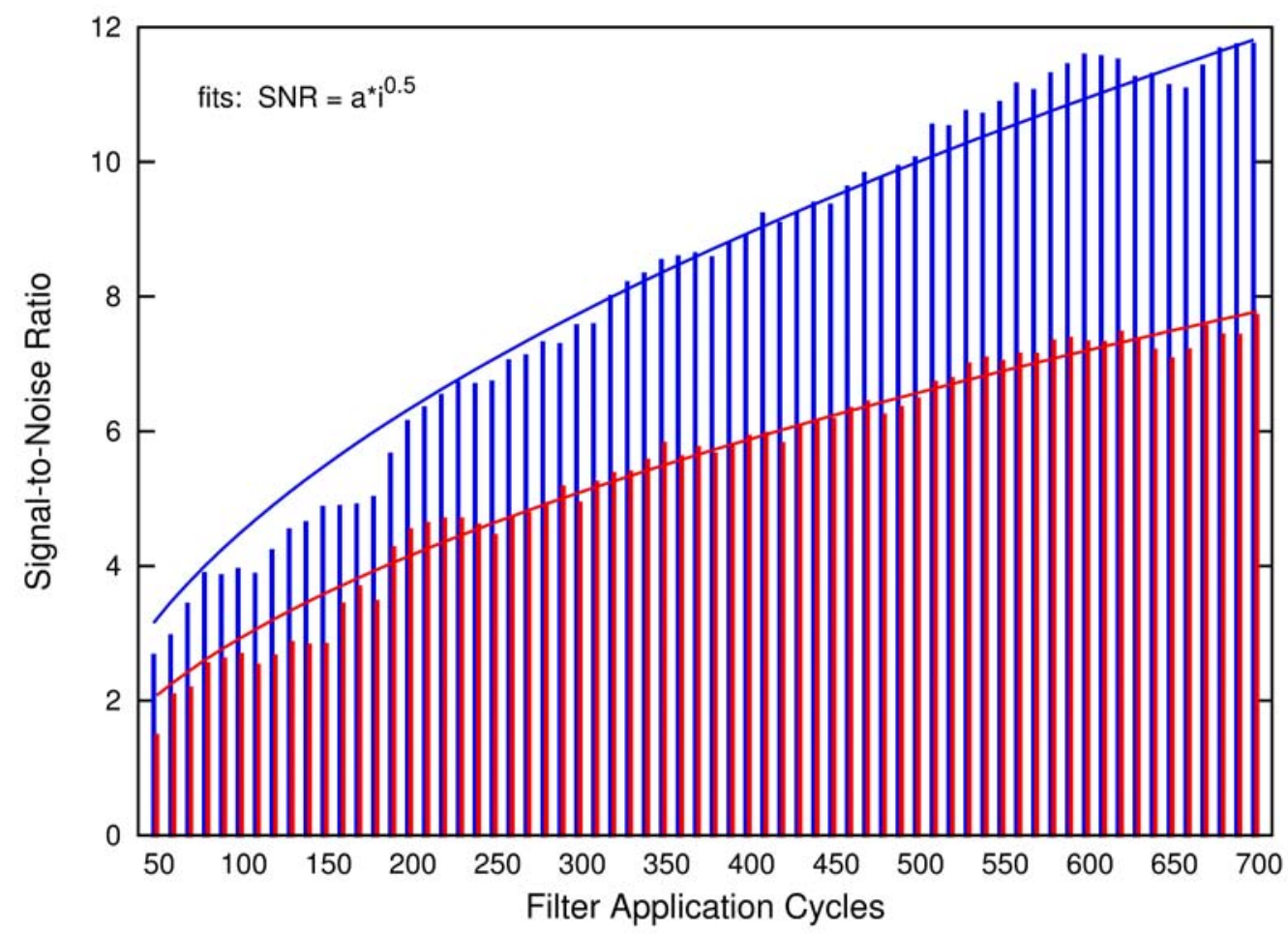

Figure 2: Example of calculated signal-to-noise ratio (SNR) values for the inner peaks of filtered (blue, upper) and unfiltered (red, lower) spectra over 1400 minutes $(i=700)$. The applied filter utilizes $\sigma=16.5$ gaussian smoothing. Fits to the equation $\mathrm{SNR}=a i^{\frac{1}{2}}$ are given by solid lines, and give $a_{\text {filtered }}=0.446377$ and $a_{\text {unfiltered }}=0.293969$, giving filter enhancement ratio $\zeta$ $=1.51845$. Deviations from the fit at lower cycle numbers are due to greater uncertainties in peak fitting at lower count times. 


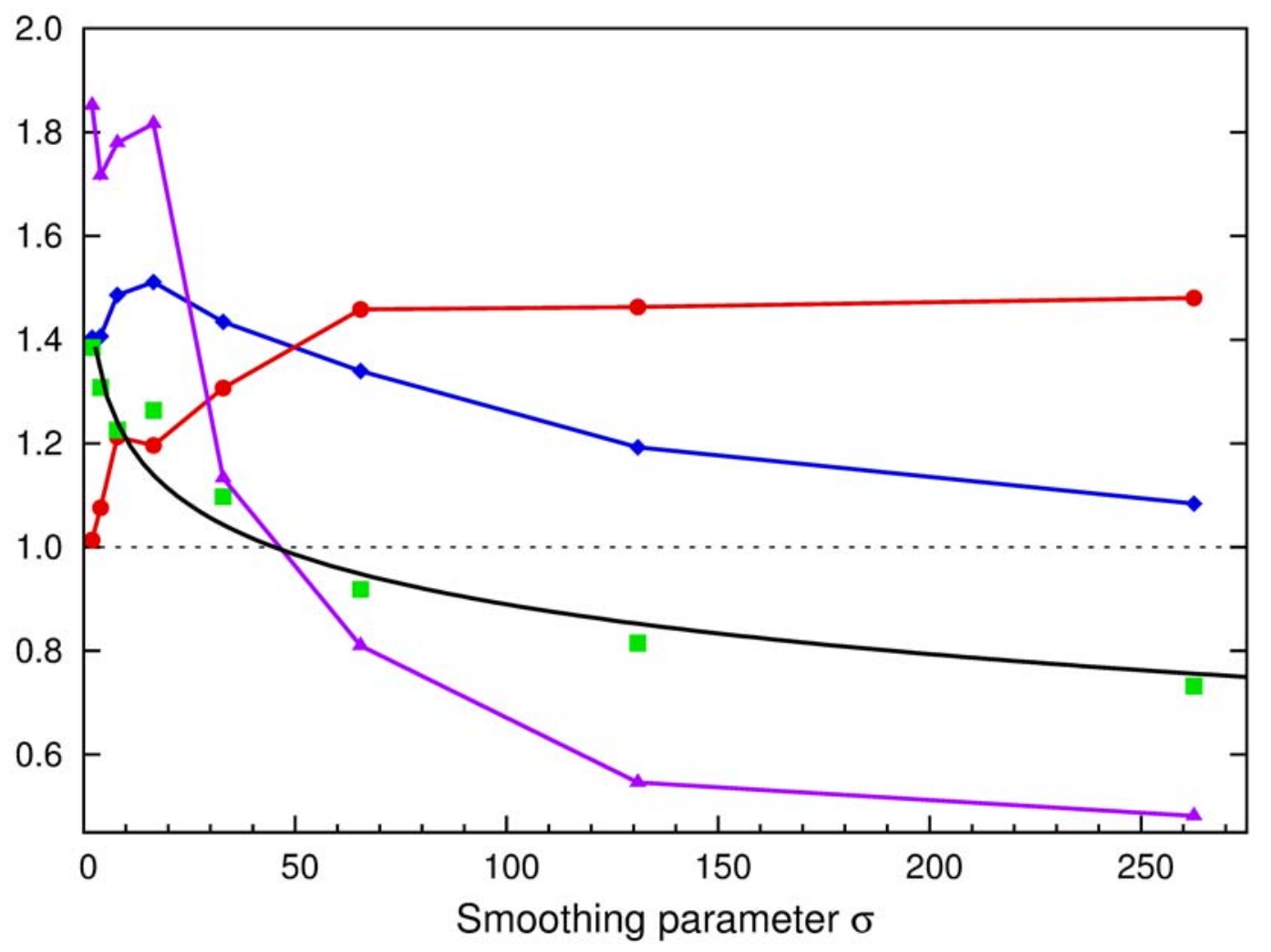

Figure 3: Signal-to-noise enhancement parameter $\zeta$ (blue diamonds), inner peak FWHM (red circles), ratio $\epsilon=\zeta /$ FWHM for filtered data (green squares), and $\epsilon$ for post-process smoothed data (purple triangles) as a function of the smoothing parameter $\sigma$. The black line corresponding to the squares represents the logarithmic fit $\epsilon=-0.138442 \ln (\sigma)+1.52729$ found for epsilon after filtering. All other lines serve as eyeguides. The value of $\epsilon$ for filtered data varies much less with $\sigma$ than $\epsilon$ for post-processed data. 